\title{
Clinicians as Secondary Users of Patient-Centered Mobile Technology in Complex Healthcare Settings
}

\author{
Harry D. Tunnell IV \\ Dept. of Human Centered Computing \\ Indiana University, School of \\ Informatics \& Computing, IUPUI \\ Indianapolis, USA \\ hadtunne@iupui.edu
}

\author{
Anthony J. Faiola \\ Dept. of Human Centered Computing \\ Indiana University, School of \\ Informatics \& Computing, IUPUI \\ Indianapolis, USA \\ faiola@iupui.edu
}

\author{
David A. Haggstrom \\ VA HSR\&D Center \\ Indiana University, School of \\ Medicine, IUPUI \\ Indianapolis, USA \\ dahaggst@iupui.edu
}

\author{
Preethi Srinivas \\ Dept. of Human Centered Computing \\ Indiana University, School of \\ Informatics \& Computing IUPUI \\ Indianapolis, USA \\ presrini@iupui.edu
}

\begin{abstract}
This paper describes the preliminary research findings and prototype development of a Personal Health Record mobile application. A pilot study about patient-clinician interaction guided by common ground theory was performed. The goal of the pilot study was to gather requirements to support development of a smartphone application to be used in a future experimental study. Findings from the pilot study suggest that smartphones could be used to manage health information considered important for a successful healthcare consultation.
\end{abstract}

Keywords-secondary user; primary user; personal health record; health information technology

\section{INTRODUCTION}

An expanding area of research has developed in the area of patient-centered health information technology (HIT). In particular, mobile technology (e.g., smartphones) have the potential to improve patient-clinician communication (in clinical settings) through the use of personal health records (PHR) [1, 2]. PHRs are patient-centered tools that people use to store, manage, and share personal healthcare data. They are important because they provide the lay person with access to personal health information that can impact patient-physician decision-making [3]. Smartphone PHR applications (apps) are of particular interest because they give patients the ability to access relevant healthcare information anywhere-anytime.

Until recently, clinicians as primary users, have been the dominant users of HIT [4]. (Clinicians refer primarily to physicians, nurses, therapists, and physician assistants, or nurse practitioners.) Conversely, patients as secondary users are those who were directly affected by the primary user's operation of the technology [4-6]. In the latter case, secondary users have often had little or no opportunity to interact with the data accessed via the technology. Patient-centered system design reverses these roles, putting the patient in the position of the primary user (Figure 1) and in control of the technology.

The Pew Research Center reported that $62 \%$ of all U.S. adults using smartphones have used them to gather health-related information [7], making them akin to an ad hoc patientcentered technology. Because smartphones are small enough to be manipulated in most environments, they have potential as a practical item in the clinical setting. Encounters with clinical staff frequently occur in small, enclosed spaces that impose limitations on the tools that can be used to enhance communication [8].
While there is a rich body of knowledge regarding primary users, the study of secondary users in the context of complex patient-clinician communication has been neglected. As such, common ground theory provides the theoretical framework for our pilot study. This paper presents the results of the pilot study, which was an initial guided requirements elicitation process for a prototype to be used in a forthcoming experimental study.

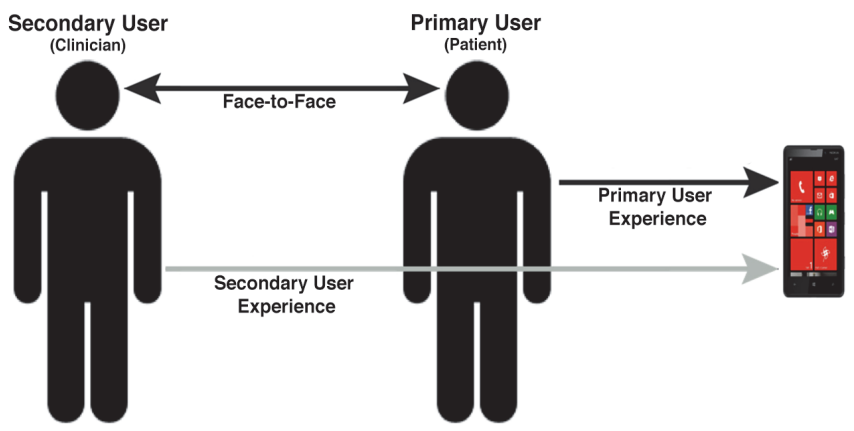

Fig. 1. Descriptive model for face-to-face interaction showing location of primary and secondary users with respect to shared technology usage.

\section{THEORETICAL FRAMEWORK}

Common ground is a communications theory that describes language use as a joint action between participants [9]. Common ground is established when people have certain knowledge in common and they know they have this common knowledge [10]. The joint action occurs when people work together to establish shared knowledge, and this process - the joint action to create common ground - is known as grounding. For example, physiotherapists seeking shared understanding related to reaching consensus and a mutually acceptable follow-up plan, both of which facilitate collaborative decision-making, are engaged in a joint action with their patients [11].

PHRs provide patients with better access to their health data. Improving patient access to their own health information leads to increased patient participation in health-related decision-making [12]. However, it is not merely the access to data that causes this impact. In reality, it is the improvement in common ground between patients and clinicians that make superior patient engagement possible. For this reason, the notion of common ground is extremely vital for exploring the role of smartphones in patient-clinician communication. 


\section{BACKGROUND}

There is a substantial body of research about primary user experiences (UXs). Consequently, they are often considered and designed for during system development. We also know that secondary users have UXs with many types of technology [13]. However, we do not understand when these experiences have a meaningful impact on user satisfaction. This is why common ground is relevant as a theoretical perspective. It makes sense that secondary users should be considered in design when joint actions for grounding are anticipated. A better understanding of secondary users, and how their communication with primary users is impacted by technology, should also be considered in human-computer interaction (HCI) design. A limited number of studies examining the secondary user experience have been done in clinical and non-clinical settings [14-16]. Examples of secondary user experiences in clinical settings have been reported in a small, but relevant number of studies.

Alsos and Svanæs [5] performed two usability studies with role-players in a hospital setting. In these studies, physicians used a mobile personal digital assistant (PDA) to access the electronic patient record system in various scenarios. The scenarios were modeled after common clinical rounds performed with patient role-players. The researchers concluded from their qualitative findings that primary (physician) user interaction with devices had a direct impact on secondary users. In summary, they concluded that UX interaction for primary and secondary users should be designed together rather than separately, i.e., devices should support non-verbal communication, provide feedback to the secondary users, and be tailored to address conflicting needs between the primary and the secondary user. Alsos, et al. [17] also conducted two experimental studies of physicians performing simulated rounds using patient records on paper, a PDA, and a laptop (on a wheeled cart). Findings suggested that secondary users need to be supported by three factors that influence the interaction between physicians and patient: the user interface, device form factor, and physician communication practice.

Finally, Gonzales and Riek [18] evaluated the notion of using a shared device to improve communication between physicians (primary users) and cancer patients (secondary users). Through interviews and contextual inquiries with oncologists, a number of unique design considerations emerged. Qualitative outcomes of the study suggested that a shared mobile device that presented tailored information to the patient's treatment plan would improve the quality of communication. They developed a concept for HIT personalized to the patient in a shared display that the physician and patient viewed simultaneously.

These studies, along with the greater domain of literature, demonstrate two key factors. First, that patients as secondary users have limited forms of UX, calling the meaningfulness of their HCI experience into question; and second, that the level and quality of communication between the two user groups is unclear. Moreover, we believe that the scenarios portrayed in these studies do not demonstrate how designing for a reversed-role UX would impact the quality of communication between the physician as secondary user and the patient as the primary user, while using mobile HIT, such as smartphones.

As such, we see the patient as the primary user of PHRs and their clinician as the secondary user: providing a novel problem space for inquiry. We argue that designing mobile HIT for both primary and secondary users will ensure a quality of communication between patient and clinician that includes enabling both users the means to successfully communicate and perform desired tasks without frustration. The scope of this paper only addresses the preliminary findings of our study leading up to and including the creation of the first mobile PHR app prototype.

\section{A. Preliminary Interviews \\ IV. Methods}

Interviews were conducted with a convenience sample of four participants: two physicians, one PHR company CEO (also a physician), and one PHR company president. Participants were identified through faculty of the Indiana University School of Informatics and Computing, Indianapolis and a non-profit board member. Participants were informally interviewed by phone and face-to-face using open-ended questions focused on current use of HIT and the future of PHR use. The focus of the interviews was to identify the HIT and PHR problem space.

\section{B. Focus Group Discussion}

A focus group session was conducted consisting of a convenience sample of 12 members of an Indiana inventors club (IIC) in a large Midwestern city, some of whom had healthcare and engineering experience. The face-to-face group discussion was facilitated by the lead author and explored PHR smartphone app design functionality and context of use. Focus group facilitator questions were informed from findings of preliminary interviews. The purpose of the focus group was to identify and confirm mobile PHR app development usability/functionality issues surrounding product integration in clinical settings.

\section{Semi-Structured Interviews}

Semi-structured interviews consisting of a convenience sample of one clinician and one potential PHR mobile app user were conducted. The interviews were audio recorded and manually transcribed. (Findings from the preliminary interviews and focus group discussions informed the design of the interview questionnaires). The purpose of the interviews was to identify and confirm design and usability issues related to mobile PHR app development, HCI, and quality of communication between the primary and secondary users.

\section{Thematic Content Analysis}

A thematic analysis of seven primary common-ground research papers and the two semi-structured interviews (as outlined above) were initially coded separately using the qualitative analysis software, NVivo 10. During each round of coding, notes were taken and included in subsequent rounds of coding. After initial coding, the literature and interview content was explored using a customized frequency word search. Text keyword searches were also performed. The keyword search showed concepts that a keyword was related to visualized in a word tree. The visualization (Figure 2) made it easy to identify keywords with high frequency. The purpose of the literature analysis was to identify common ground themes that could be operationalized into design constructs. The purpose of the semistructured interview content analysis was to identify critical elements of the patient-clinician interaction and how each manages information. 


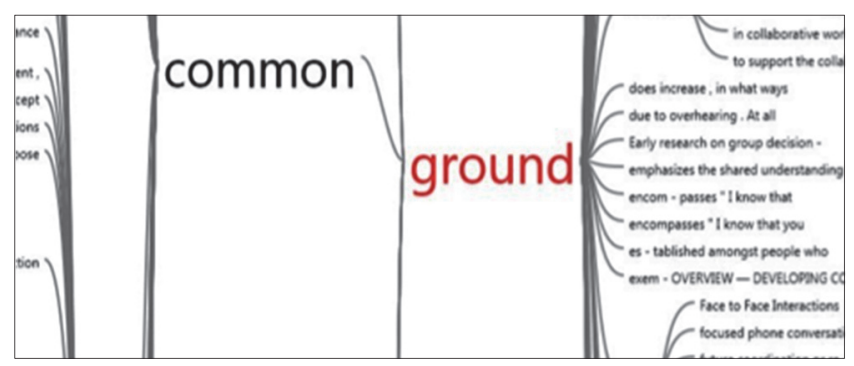

Fig. 2. A visual example of the output of the text keyword search for ground.

\section{REsults}

Findings from the preliminary interviews regarding patientclinician interaction highlighted several critical topics of communication during an interaction. A summary of the interview findings is that there should be patient-clinician shared/agreed understanding of: (1) the medication list, (2) allergy information (i.e., food and medication), (3) past medical history, and (4) present illness and treatment plan. Findings from the focus group discussion identified concurrence on two issues: (1) sharing between primary and secondary users must be simple while remaining within the constraints of privacy and security policies; and (2) consider wireless sharing of patient information with the provider's computing device. A summary of the findings from the semi-structured interviews revealed: (1) patients do not specifically prepare for an appointment and believe they have sufficient knowledge to tell the doctor what is going on, (2) patients lack detailed knowledge for the appointment, and (3) doctors want any data shared with them electronically by the patient to be compatible with the EHR - so they can look at a similar data view.

The thematic content analysis ultimately yielded three final themes: content and process knowledge, shared understanding, and collaboration is limited by a lack of interactivity. Interestingly, the semi-structured interview part of the content analysis highlighted tension between patients and clinicians about the quality of patient-clinician communication. For example, patients believed that they should provide quality information to clinicians, while clinicians believed the opposite.

"When it's my own personal health I don't really feel that I need, in the context of an appointment, information [i.e., medical history] on an app, for example, I can just communicate and tell the doctor directly." - Patient Interviewee

"Most patients will refer to the computer. So if you said what medications are you taking or what medical problems do you have... they'll basically say 'it's in the computer' or 'refer to an external resource." - Clinician Interviewee

\section{Prototype Design AND Testing}

\section{A. Paper Prototyping}

Aggregated findings informed four iterations of sketching for the mobile app interfaces, followed by three iterations of low-fidelity paper-in-screen prototypes [19]. The paper-inscreen prototyping technique (Figure 3 ) allows paper sketches to be uploaded into a smartphone to better simulate user interaction. A usability evaluation, using cognitive walkthroughs [20], was performed on the paper-in-screen prototypes. Two IIC members were recruited as participants for the cognitive walkthrough testing. Specific usability problems were identified, recorded, and addressed during medium-fidelity prototyping.

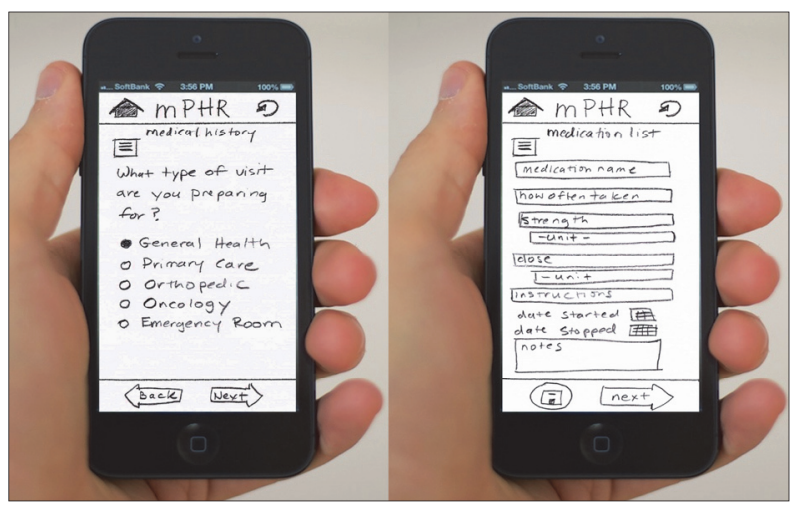

Fig. 3. Two examples of paper-in-screen prototyping technique.

\section{B. Medium- and High-Fidelity Prototyping}

A medium-fidelity prototype in wireframe format was created (using Adobe Fireworks). An inspection of the wireframe revealed additional usability problems, which were addressed during a subsequent design iteration (e.g., a consolidation of repetitive categories on the menu, additional warnings for sharing information electronically, and customized output screens for different user types). From these revisions, an interactive high-fidelity prototype in HTML (also in Adobe Fireworks) was created. (See Figure 4.)

Design aspects identified as indispensable for enabling the grounding experience were successfully represented in the prototype. For example, the clinician interview indicated that different views of information for primary and secondary users are a suitable technique for improving communication. For instance, the problem list interface (identified during our data collection process) was originally organized with one output screen, primarily with textual information. To address the clinician's perspective, it was revised to include multiple output screens (Figure 4): one with less text, the addition of an icon, and an additional screen with output organized similar to an electronic health record (EHR), which is the system that clinician's use.

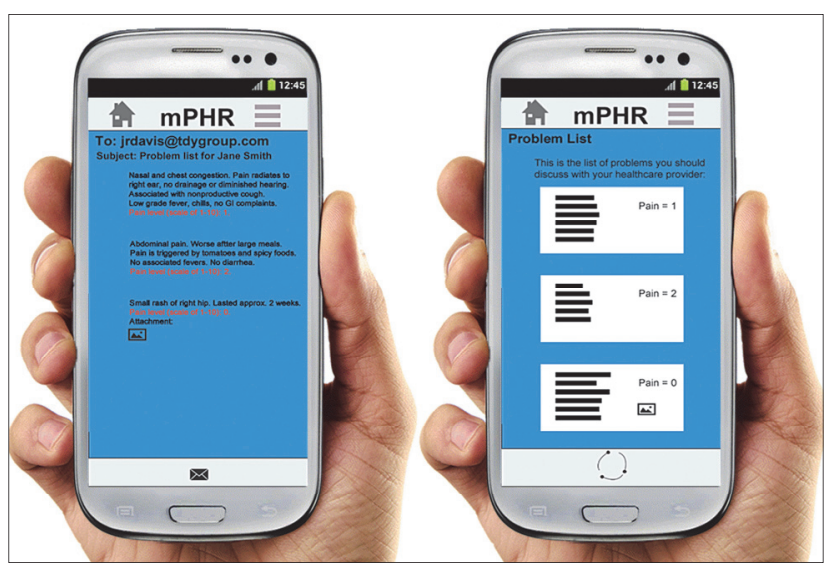

Fig. 4. (L) Problem list output organized for clinicians, (R) Problem list output organized for patients. 


\section{DISCUSSION}

The pilot study identified findings that suggest a possible disparity in perceptions of knowledge about patient health information between patients and clinicians. If confirmed, this means that there is a lack of grounding between them. Our interviews revealed that most of the tools to ensure that common ground is attained are manual (e.g., active listening) and (often times) the responsibility of the clinician. This places the primary burden of communication on the clinician. The patient, however, has the most personal knowledge about how they feel and their overall health status at the time of engagement with a clinician. As such, mobile HIT in the form of PHR apps can significantly improve this communication. That is to say, the results from such HIT-facilitated encounters have the potential to push the traditional boundaries of interaction by enhancing the quality of the exchange.

The findings of the pilot study demonstrate that smartphones are able to support the type of patient health information appropriate for a successful healthcare consultation. It also demonstrated that gaps likely exist in patient knowledge necessary for grounding. Thus, smartphone PHR apps have the potential to improve the quality of communication by enhancing accuracy and speed of information sharing between patients and clinicians, which we believe is imperative to overcoming breakdowns of understanding during an attempted joint action. Challenges for future research include identifying and addressing user concerns about privacy and security.

Several insights were obtained from the prototype iterations, and our continuing research, which have yielded a rich data source about primary and secondary users. We observed that there continues to be a disparity in knowledge between clinician and patient regarding the patient's health status. At the same time, clinicians were not confident that current HIT could reliably support a transfer of patient-shared PHR data to the clinician's EHR during a real-time health consultation.

\section{FUTURE WORK}

While the outcomes of our pilot study have resulted in several key "lessons learned" on patient-clinician communication, other forms of interaction (e.g., email or SMS) cannot sustain the level of detail of information that can be distributed (in real-time) via the smartphone PHR app proposed here. Moreover, the findings of this pilot study have already proven important for the final prototype requirements elicitation process that is on-going. For example, an online survey will be developed to validate and extend what was learned during the pilot study and follow-on patient and clinician interviews. The result will be enhanced design guidelines to better inform app prototype development and the forthcoming experimental study. Hence, the prototype design and usability study will continue to focus on the simultaneous primary and secondary UXs.

In summary, to our knowledge, this will be the first patient-centered mobile HIT design that embraces a synchronized UX for both user-types. And as such, we anticipate that they will be equally benefited from the final prototype design, development, and usability evaluation phases.

\section{REFERENCES}

[1] J. P. Weiner, "Doctor-patient communication in the e-health era," Israel Journal of Health Policy Research, vol. 1, 2012.

[2] K. M. Nazi, "The personal health record paradox: Health care professionals' perspectives and the information ecology of personal health record systems in organizational and clinical settings," Journal of Medical Internet Research, vol. 15, 2013.

[3] L. Bos, A. Marsh, D. Carroll, S. Gupta, and M. Rees, "Patient 2.0 Empowerment," in Conference on Semantic Web \& Web Services, 2008, pp. 164-168.

[4] K. Eason, Information technology and organizational change. New York, NY: Taylor \& Francis, 1988.

[5] O. A. Alsos and D. Svanæs, "Designing for the secondary user experience," presented at the 13th International Conference on Human-Computer Interaction, Lisbon, Portugal, 2011.

[6] C. Abras, D. Maloney-Krichmar, and J. Preece, "User-centered design," in Encyclopedia of Human-Computer Interaction. vol. 37, W. Bainbridge, Ed., ed Thousand Oaks: Sage Publications, 2004, pp. 445-56.

[7] A. Smith and D. Page, "The smartphone difference," Pew Research Center, Washington, DC2015.

[8] M. J. Gonzales, M. F. O'Connor, and L. D. Riek, "Contextual constraints for the design of patient-centered health IT tools," Context Sensitive Health Informatics: Human and Sociotechnical Approaches, vol. 194, pp. 75-81, 2013.

[9] H. H. Clark, Using language New York, NY: Cambridge University Press, 1996.

[10] W. A. Kellogg, T. Erickson, T. V. Wolf, S. Levy, J. Christensen, J. Sussman, et al., "Leveraging digital backchannels to enhance user experience in electronically mediated communication," presented at the 20th Anniversary Conference on Computer Supported Cooperative Work, Banff, Alberta, Canada, 2006.

[11] M. C. Politi and R. L. S. Jr., "The importance of communication in collaborative decision making: facilitating shared mind and the management of uncertainty," Journal of Evaluation in Clinical Practice, vol. 17, pp. 579-584, 2010.

[12] J. E. Prey, J. Woollen, L. Wilcox, A. D. Sackeim, G. Hripcsak, S. Bakken, et al., Patient engagement in the inpatient setting: A systematic review vol. 21, 2014.

[13] E. Montague, "Understanding patient user experience in obstetric work systems," in Ergonomics and Health Aspects of Work with Computers. vol. 5624, B.-T. Karsh, Ed., ed: Springer Berlin Heidelberg, 2009, pp. 7077.

[14] M. Berg, C. Langenberg, I. vd Berg, and J. Kwakkernaat, "Considerations for sociotechnical design: Experiences with an electronic patient record in a clinical context," International Journal of Medical Informatics, vol. 52, pp. 243-251, 1998.

[15] H. Huttenrauch, A. Green, M. Norman, L. Oestreicher, and K. S. Eklundh, "Involving users in the design of a mobile office robot," IEEE Transactions on Systems, Man, and Cybernetics, Part C: Applications and Reviews, vol. 34, pp. 113-124, 2004.

[16] E. Moschini, "Designing for the smart player: Usability design and usercentred design in game-based learning," Digital Creativity, vol. 17, pp. 140-147, 2006.

[17] O. A. Alsos, A. Dasb, and D. Svanæs, "Mobile health IT: The effect of user interface and form factor on doctor-patient communication," International Journal of Medical Informatics, vol. 81, pp. 12-28, 2012.

[18] M. J. Gonzales and L. D. Riek, "A shared interface to improve oncologist-patient communication," in 6th International Conference on Pervasive Computing Technologies for Healthcare, San Diego, CA, 2012.

[19] D. Bolchini, D. Pulido, and A. Faiola. (2009) Paper in screen prototyping: An agile technique to anticipate the mobile experience. Interactions. 2933.

[20] D. Pinelle and C. Gutwin, "Groupware walkthrough: Adding context to groupware usability evaluation," in SIGCHI Conference on Human Factors in Computing Systems: Changing our World, Changing Ourselves, 2002, pp. $455-462$. 